\title{
A Universal Frequency Offset Estimator for OFDM Applications
}

\author{
Ming (Matt) Ruan, Mark C. Reed \\ National ICT Australia \\ ACT, Australia \\ Email: $\{$ matt.ruan, mark.reed\}@ nicta.com.au
}

\author{
Zhenning Shi \\ Alcatel Shanghai Bell \\ Shanghai, China \\ Email: Zhenning.Shi@alcatel-sbell.com.cn
}

\begin{abstract}
This paper studies fractional carrier frequency offset (CFO) estimation for orthogonal frequency-division multiplexing (OFDM) systems. In the IEEE 802.16 (WiMAX) standard, a training symbol structure with three highly correlated but not identical segments is specified. The existing CFO estimation methods require the segments in the training symbol to be identical, so cannot work for the generalized training structure. In this paper, we proposed a universal fractional CFO estimator to solve the problem. Both the analytical and numerical results are presented to confirm the performance of the proposed method.
\end{abstract}

Index Terms-Orthogonal frequency-division multiplexing (OFDM), synchronization.

\section{INTRODUCTION}

Orthogonal frequency-division multiplexing (OFDM) has been adopted by many wireless communication standards to provide broadband access in frequency selective wireless channels. Because the orthogonality of the transmission relies on the frequency synchronization between the transceivers, carrier frequency offset (CFO) will cause dramatic performance degradation.

In packet oriented OFDM systems, synchronization and channel estimation are facilitated by the transmission of training symbols at the beginning of each packet. In some practical OFDM systems like the IEEE 802.16 [1], the training symbol is defined in the frequency domain by using one out of every $M$ subcarriers, where $M$ is the interval between every two used subcarriers. When $M$ is not divisible by the number of subcarriers, e.g., $M=3$ for the downlink of IEEE 802.16 OFDMA (WiMAX) system, the training symbol is made up of highly correlated but not identical segments in the time domain. This scenario has not been investigated by the existing literature [2]-[6], and Bhatt et al [7] suggested to use the cyclic prefix for frequency estimation.

In this paper, we propose a universal fractional CFO estimator for the generic WiMAX training symbols. When the training symbol has a perfect repetitive structure, the proposed method outperforms the extended Schimidl-Cox algorithm (ESCA) [5] at low SNR, and is only $0.51 \mathrm{~dB}$ away from the Cramer-Rao bound (CRB) at high SNR. Existing methods are not applicable to the scenario where the segments of the training symbol are not identical, while the proposed method still approaches the CRB within $1.1 \mathrm{~dB}$ loss for all $M \in[2,16]$. It is also possible to use the method of [5] to combine the estimates according to the best linear unbiased estimator (BLUE) principle. We derive the BLUE coefficients for the proposed universal estimators, and show that the marginal performance improvement may not be able to justify the extra complexity.

This paper is organized as follows. We define the system model in Section II, then derive a universal fractional CFO estimator and the BLUE based on this universal estimator in Section III. In Section IV, the performance of the proposed methods are compared against the CRB. Simulation results obtained with realistic channel models are presented in Section V and a brief summary concludes the paper in Section VI.

\section{SySTEM MOdEL}

We study packet based OFDM systems where a training symbols precedes every packet to facilitate timing, frequency and channel estimation. The time-domain representation of the training symbol in the time duration $t \in\left[0,\left(N+N_{g}\right) T_{s}\right]$ can be written as

$$
x(t)=\frac{1}{\sqrt{N}} \sum_{m=1}^{N_{p}} X[m] e^{-j \frac{2 \pi}{N}\left(\vartheta_{0}+(m-1) M\right)\left(t / T_{s}-N_{g}\right)}
$$

where $N$ is the number of subcarriers; $T_{s}$ is $(1 / N)$-th of the symbol duration, which is also known as the duration of one OFDM sample; $N_{g}$ is the length of cyclic prefix (CP) in terms of samples; $N_{p}$ is the number of used subcarriers in the training symbol, and the index for the first used subcarrier is $\vartheta_{0} ; M$ is the interval between every two used subcarriers; $X[\cdot]$ is a phase shift keying (PSK) modulated pseudo-random training sequence. In some practical OFDM systems, the base stations may use different $\vartheta_{0}$ to mitigate inter-cell interference, so perfect knowledge about $\vartheta_{0}$ is not available to the receiver.

Assume the baseband signal $x(t)$ passes through a low-pass filter and is sampled at time interval $T_{s}$. We obtain a sequence of samples $x[n]=x\left(n T_{s}\right)$. If $N$ is not an integer multiple of $M$, the period of $x(t)$ is not sample-spaced, then the repetition structure is not preserved in the sampled sequence $x[n]$. For instance, if $N=128$ and $M=3$, one period of $x(t)$ is $\frac{128}{3} T_{s}$, hence $x[1]$ is not identical to either $x[42]$ or $x[43]$, but they are all highly correlated. 
After low-pass filtering and decimation, the equivalent baseband received signal writes

$$
\begin{aligned}
r[n] & =e^{j \frac{2 \pi}{N} n \epsilon_{0}}\left(\sum_{l=1}^{L_{h}} h[l] x[n-\tau[l]]+w[n]\right) \\
& =e^{j \frac{2 \pi}{N} n \epsilon_{0}}(\hat{x}[n]+w[n])
\end{aligned}
$$

where $\epsilon_{0}$ is the true CFO normalized by one subcarrier spacing $1 /\left(N T_{s}\right) ; h[l]$ is the complex multiplicative channel gain of the $l^{\text {th }}$ path whose delay is $\tau[l] ; w[n]$ is the additive white Gaussian noise (AWGN) with variance $\sigma_{w}^{2}$; and, we define $\hat{x}[n]=\sum_{l=1}^{L_{h}} h[l] x[n-\tau[l]]$. In this paper, signal to noise ratio (SNR) is defined as the ratio between the total received signal and noise power in the training symbol, i.e., $\mathrm{SNR} \triangleq\left(\sigma_{s}^{2} / \sigma_{w}^{2}\right)$, where $\sigma_{s}^{2}=\frac{1}{N} \sum_{k=N_{g}}^{N+N_{g}-1}\left|\hat{x}\left[(i-1)\left(N+N_{g}\right)+k\right]\right|^{2}$.

\section{THE METHOD}

\section{A. Correlators}

We assume coarse timing estimate $\hat{n}$ is available and it does not incur any inter-symbol interference. Following the approach of conventional methods [2]-[6], we construct CFO estimators based on the correlators, which are defined as

$$
\begin{aligned}
R(d) & \triangleq \sum_{k=0}^{N-d} r^{*}[\hat{n}+k] r[\hat{n}+k+d] \\
& \approx e^{j \frac{2 \pi}{N} d \epsilon_{0}}\left(\sum_{k=0}^{N-d-1} \hat{x}^{*}[\hat{n}+k] \hat{x}[\hat{n}+k+d]+\Theta(d)\right)
\end{aligned}
$$

where the approximation neglects the products of uncorrelated noise and

$\Theta(d)=\sum_{k=0}^{N-d-1} w^{*}[\hat{n}+k] \hat{x}[\hat{n}+k+d]+\sum_{k=d}^{N-1} w[\hat{n}+k] \hat{x}^{*}[\hat{n}+k-d]$.

As shown in [8], when the signal is not weaker than the noise, the correlation output can be approximated by

$$
R(d) \approx e^{j \frac{2 \pi}{N} \epsilon_{0} d}\left((N-d) \sigma_{s}^{2} \phi(d) \rho(d)+\Theta(d)\right)
$$

where

$\rho(d)= \begin{cases}1, & d \text { is a multiple of }(N / M) ; \\ \frac{\sin \left(\frac{\pi}{N} M d N_{p}\right)}{N_{p} \sin \left(\frac{\pi}{N} M d\right)}, & \text { others. }\end{cases}$

$\phi(d)= \begin{cases}e^{j \frac{2 \pi}{N} \vartheta_{0} d}, & d \text { is a multiple of }(N / M) ; \\ e^{j \frac{\pi}{N} d\left(M\left(N_{p}-1\right)+2 \vartheta_{0}\right)}, & \text { others. }\end{cases}$

Because only the first term in the bracket on the right hand side of (5) contains useful information about CFO, $d$ should be designed to be close to a multiple of $N / d$ to maximize the magnitude of the useful signal.

Conventional methods [2]-[6] assume both $d$ and $\vartheta_{0}$ are multiples of $(N / M)$ and estimate CFO from the phase of $R(d)$ as

$$
\hat{\epsilon}(d)=\frac{N}{2 \pi d} \arg \left(R(d) \cdot e^{j 2 \pi m}\right)=\frac{N}{2 \pi d} \arg (R(d))+\frac{N}{d} m,
$$

where $m$ can be any integer. A usable fractional CFO estimator should give the same estimate for all $m$. For instance, when $N$ is a multiple of $d$, the second term on the right hand side of (8) is an integer, therefore for all $m$,

$$
[\hat{\epsilon}(d)]_{1}=\frac{N}{2 \pi d} \arg (R(d))
$$

where $[\cdot]_{1}$ represents the modulo- 1 function that only takes the fractional part of its argument. This means that conventional methods will not have the fractional ambiguity problem when $N$ is divisible by $M$.

However, when $(N / d)$ is not an integer, the second term on the right hand side of (8) also has a fractional component, which will cause large estimation errors in the fractional CFO estimates. Many integer CFO estimators like the one proposed in [9] work on the frequency domain signal. The residual fractional CFO error that is not compensated in the time domain will compromise the performance of those frequencydomain signal processing algorithms.

\section{B. Universal CFO Estimators}

From the analysis above, we know the CFO estimates based on a single correlator has an ambiguous fractional part when $(N / d)$ is not an integer. To solve the problem, we multiply $R(d)$ with another complementary term such that the second term on the right hand side of (8) becomes an integer for all $m$. The complementary term universal to all $d$ values is found to be $R(N-d)$, and the CFO can be estimated by

$$
\hat{\epsilon}\left(d_{k}\right)=\frac{1}{2 \pi} \arg \left(R\left(d_{k}\right) R\left(N-d_{k}\right)\right)
$$

where $d_{k}$ is the correlation interval which should be close to a multiple of $(N / M)$. Multiple universal CFO estimators can be constructed for one training symbol. They have the same estimation range of \pm 0.5 subcarrier spacing, but different estimation performance. Assume $\hat{\epsilon}\left(d_{o}\right)$ has the minimum mean square error (MSE) among the universal estimators, we refer to $\hat{\epsilon}\left(d_{o}\right)$ as the best universal estimator (BUE).

In Appendix, we show that when the estimation error is small,

$$
E\left\{\hat{\epsilon}\left(d_{k}\right)\right\}=\left[\epsilon_{0}\right]_{1},
$$

so the universal CFO estimators are unbiased. Also, the MSE of the universal estimates is shown to be

$$
\begin{aligned}
& E\left\{\left(\hat{\epsilon}\left(d_{k}\right)-\left[\epsilon_{0}\right]_{1}\right)^{2}\right\}=\frac{N\left(N-d_{k}\left(1-\rho\left(2 d_{k}\right)\right)\right)}{4 \pi^{2} \rho_{i}^{2}\left(d_{k}\right) d_{k}\left(N-d_{k}\right)^{2}} \frac{\sigma_{w}^{2}}{\sigma_{i}^{2}} \\
= & \frac{N^{2}}{4 \pi^{2} d_{k}\left(N-d_{k}\right)^{2}} \mathrm{SNR}^{-1} \cdot \eta\left(d_{k}\right)
\end{aligned}
$$

where

$$
\eta\left(d_{k}\right)=\frac{\left(N-d_{k}\right)+d_{k} \rho\left(2 d_{k}\right)}{N \rho^{2}\left(d_{k}\right)} \geq 1 .
$$

The equality of (13) holds when $\rho\left(d_{k}\right)=1$, which requires $d_{k}$ to be a multiple of $(N / M)$; otherwise, $\eta\left(d_{k}\right)$ represents the 
performance loss caused by imperfect repetition and sampling. Using (12), one can determine the BUE as

$$
d_{o}=\underset{d}{\arg \min } E\left\{\left(\hat{\epsilon}(d)-\left[\epsilon_{0}\right]_{1}\right)^{2}\right\}=\underset{d}{\arg \min } \frac{\eta(d)}{d(N-d)^{2}} .
$$

When $M$ is divisible by $N, d_{o}=[M / 3] \cdot N / M$ where $[\cdot]$ denotes integer rounding.

For all $M$ divisible by $N$, the Cramer-Rao bound (CRB) is given in [5] as

$$
\mathrm{CRB}_{M}=\frac{3}{2 \pi^{2} N\left(1-M^{-2}\right)} \mathrm{SNR}^{-1} .
$$

When $M$ is not divisible by $N$, ideally it is possible to oversample the received signal and achieve the performance given by (15), so the CRB is applicable to those cases as well. As $M$ increases, the $\mathrm{CRB}$ approaches the limit $\mathrm{CRB}_{\infty}=\frac{3}{2 \pi^{2} N} \mathrm{SNR}^{-1}$. Compare the MSE of the BUE given by (12) and the CRBs, and neglect the imperfect sampling loss $\left(\eta\left(d_{k}\right)=1\right)$, we find

- If $N$ is multiple of $(M=3)$, the proposed CFO estimator achieves $\mathrm{CRB}_{3}$.

- As the number of identical segments increases, the MSE of the BUE asymptotically approaches the lower bound $\mathrm{CRB}_{3}$, which is only $0.51 \mathrm{~dB}$ away from $\mathrm{CRB}_{\infty}$.

- When $N$ is a power of 2, the MSE of the BUE is upper bounded by $\mathrm{CRB}_{4}$, which is about $0.73 \mathrm{~dB}$ away from $\mathrm{CRB}_{\infty}$.

\section{BLUE}

We construct multiple universal estimators by a set of $\left\{d_{k}\right\}$ which contains the integers around all the multiples of $(N / M)$. For a training symbol with $M$ highly correlated segments, there are at most $2\lfloor M / 2\rfloor$ such $d_{k}$, i.e., $d_{2 k-1}=\lfloor k \cdot N / M\rfloor$, $d_{2 k}=\lceil k \cdot N / M\rceil$, where $\lfloor\cdot\rfloor$ and $\lceil\cdot\rceil$ denote integer flooring and ceiling functions respectively, and $k \in[1,\lfloor M / 2\rfloor]$. After removing the duplicated $d_{k}$ in the sequence, we obtain $K$ different universal estimators. The minimum value of $K$ is $\lfloor M / 2\rfloor$ as in the case when $M$ is divisible by $N$. Write $\left\{\hat{\epsilon}\left(d_{k}\right)\right\}$ into a vertical vector $\hat{\boldsymbol{\epsilon}}$. Denote $\mathbf{1}$ as a vector with all one elements and of the same size as $\hat{\epsilon}$. The universal $\mathrm{CFO}$ estimates can be combined by the BLUE as [10]

$$
\hat{\epsilon}_{f}=\boldsymbol{c}^{T} \hat{\boldsymbol{\epsilon}}
$$

where

$$
c=\frac{C^{-1} \mathbf{1}}{\mathbf{1}^{T} C^{-1} \mathbf{1}}
$$

and $C$ is the estimation error correlation matrix. Note that although ESCA [5] also combines multiple CFO estimates, they are not based on the proposed universal estimators, so their results are not applicable to the BLUE in this paper. As shown in Appendix, the elements of the error correlation matrix are given by

$$
\begin{aligned}
C\left[k_{1}, k_{2}\right]= & E\left\{\left(\hat{\epsilon}\left(d_{k_{1}}\right)-\left[\epsilon_{0}\right]_{1}\right)\left(\hat{\epsilon}\left(d_{k_{2}}\right)-\left[\epsilon_{0}\right]_{1}\right)\right\} \\
= & \frac{N}{4 \pi^{2}} \frac{\sigma_{w}^{2}}{\sigma_{s}^{2}} \frac{1}{\rho\left(d_{k_{1}}\right) \rho\left(d_{k_{2}}\right)}\left(\frac{\rho\left(d_{k_{1}}+d_{k_{2}}\right)}{\left(N-d_{k_{1}}\right)\left(N-d_{k_{2}}\right)}\right. \\
& \left.+\frac{\rho\left(d_{k_{1}}-d_{k_{2}}\right)}{\left(N-\min \left(d_{k_{1}}, d_{k_{2}}\right)\right) \max \left(d_{k_{1}}, d_{k_{2}}\right)}\right) .
\end{aligned}
$$

The BLUE coefficient $c$ can be pre-calculated without SNR knowledge because the noise variance $\sigma_{w}^{2}$ in (18) does not change with $k_{1}$ and $k_{2}$ and will be canceled out.

\section{Summary}

Conventional CFO estimators only use one correlator [2][4] or combine multiple correlators for an extended estimation range [5], [6]. When the training symbol does not consist of identical repetitive segments, conventional estimators cannot solve the ambiguity in fractional CFO estimates. The proposed universal estimator solves the problem by combining the phases of a pair of specially constructed correlators. To maximize cost-effectiveness, one can employ the best universal estimator (BUE) to achieve the best performance with minimum complexity. To maximize performance, one can combine a number of universal CFO estimators using the BLUE principle based on the error correlation matrix (18).

\section{Performance Analysis}

In this section we analyze the performance of the proposed fractional CFO estimators. The MSE of the BUE is given by (12), and that of the BLUE based on the universal estimator can be written as [10]

$$
E\left\{\left(\hat{\epsilon}_{f}-\left[\epsilon_{0}\right]_{1}\right)^{2}\right\}=\left(\mathbf{1}^{T} \boldsymbol{C}^{-1} \mathbf{1}\right)^{-1},
$$

where the elements of $C$ is given by (18). In the following, we numerically evaluate the MSE in (19) and compare it to that of the BUE. A typical 512-subcarrier OFDM system in AWGN channel is used in both analysis and simulation. The CFO is modeled by a uniformly distributed random variable within \pm 20 subcarrier spacing. We assume the timing estimation is perfect, and compute the analytical curves for the proposed BUE and BLUE from (12) and (19) respectively.

First, we plot the mean square error as a function of SNR in Figure 1. The results show that our analysis is quite accurate for the SNR above OdB. For lower SNR, Coulson's analytical result in [4] for the $(M=2)$ case serves as an theoretical upper bound on the error variance. In both $(M=8)$ and $(M=5)$ cases, the performance of the proposed BUE approaches that of the BLUE in a wide SNR region from $-10 \mathrm{~dB}$ to $20 \mathrm{~dB}$. The ESCA does not work for the $(M=5)$ case, and has considerably inferior performance to that of the BUE and BLUE at low SNR in the $(M=8)$ case. It should be noted that at low SNR the performance of the ESCA is even worse than the $(M=2)$ Coulson bound, which means that the linear combining procedure of the ESCA fails to offer performance improvement in the presence of high noise. In contrast, the proposed algorithms are much more robust in this scenario. 

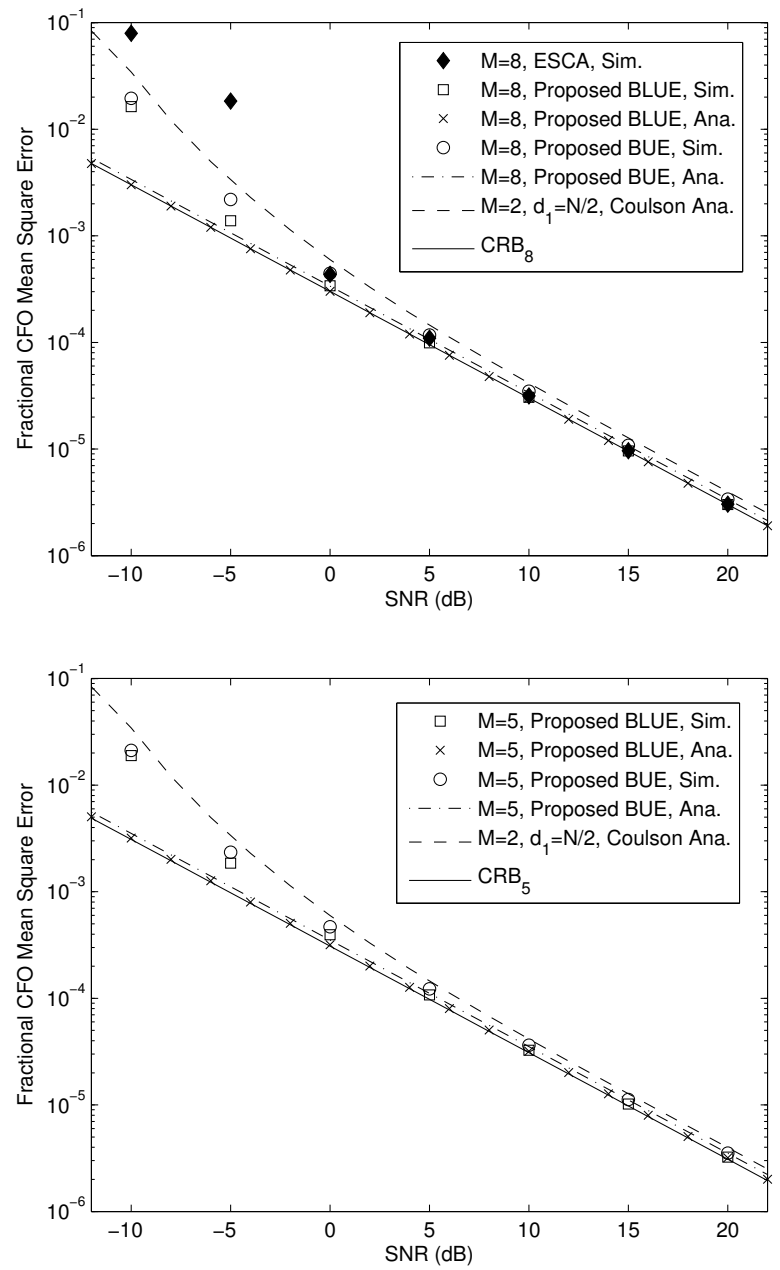

Fig. 1. Performance of the proposed fractional CFO estimation methods in AWGN channel.

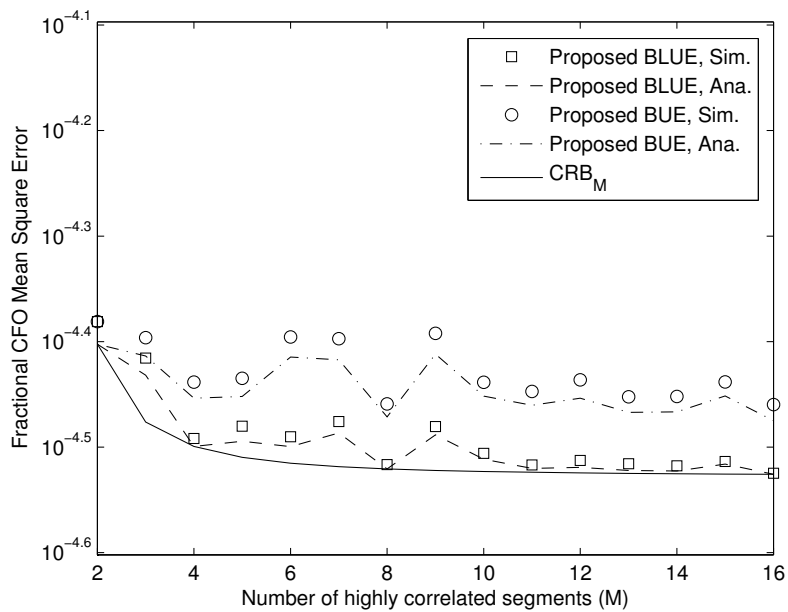

Fig. 2. The performance of the proposed schemes as a function of $L$ in one training symbol case $(\mathrm{SNR}=10 \mathrm{~dB})$.
Next, we fix the SNR to $10 \mathrm{~dB}$ and let the number of highly correlated segments $M$ change from 2 to 16 . Because both the mean square error and CRB are inversely proportional to SNR, we expect the curves remain the same except for an offset on the $y$-axis for other SNR settings. The simulation results agree with our analytical curves reasonably well in Figure 2. It is shown that the proposed BLUE can achieve CRB when $M$ is divisible by $N$, but suffers from a slight performance degradation in other cases due to the imperfect repetition and sampling. The performance loss is less than $0.44 \mathrm{~dB}$ and $1.10 \mathrm{~dB}$ for the BLUE and BUE respectively.

The proposed BUE requires two correlators except for the case of $(M=2)$ when the BUE reduces to the Schmidl's estimator [2]. The number of correlators required by the ESCA and BLUE are at least $\lfloor M / 2\rfloor$, so their complexity is much higher than that of the BUE for large $M$ values. This means that the complexity of the BLUE can be much higher than that of the BUE, while the performance improvement is marginal.

\section{Simulation Results}

An IEEE 802.16 [1] system is modeled in the simulations using the training symbol specified for the OFDMA physical layer. We use $N=512$ subcarriers and the $\mathrm{CP}$ is $1 / 8$ of one symbol duration. The timing offset estimation error is modeled by a random variable uniformly distributed within $\left[-N_{g} / 2,0\right]$ samples. A stationary wireless communication channel known as SUI-3 [11] is used in the simulations. The channel has 3 taps with relative delays $\{0,0.4 \mu \mathrm{s}, 0.9 \mu \mathrm{s}\}$ and power $\{0 \mathrm{~dB},-5 \mathrm{~dB},-10 \mathrm{~dB}\}$. The gain of the first channel tap follows Rice distribution, and those of the other two taps are Rayleigh distributed. The true CFO is modeled by a uniformly distributed random variable within \pm 20 subcarrier spacing. Every point in the figure corresponds to at least $10^{4}$ independent experiments. Because the training symbol consists of three highly correlated but not identical segments, the existing training-symbol based methods are not applicable to this scenario and Bhatt et al [7] proposed to use CP for CFO estimation. The $\mathrm{CP}$ based algorithm requires one correlator, the BUE and BLUE require two and four respectively. The simulation results in Figure 3 show that the proposed BUE outperforms the CP based method by $4 \mathrm{~dB}$, and saves half of the complexity of the BLUE at the cost of $0.3 \mathrm{~dB}$ performance loss. This illustrates the cost-effectiveness of the proposed universal $\mathrm{CFO}$ estimator.

\section{CONCLUSions}

We have proposed a universal fractional CFO estimator for the training symbols consisting of highly correlated but not necessarily identical segments. The method approaches the Cramer-Rao bound and is more robust to noise than the ESCA [5]. The BLUE based on the universal fractional CFO estimators are also derived, however, the extra complexity may not justify the marginal performance improvement.

\section{ACKNOWLEDGEMENT}

Ming (Matt) Ruan and Mark C. Reed are with the National ICT Australia and affiliated with the Australian National 


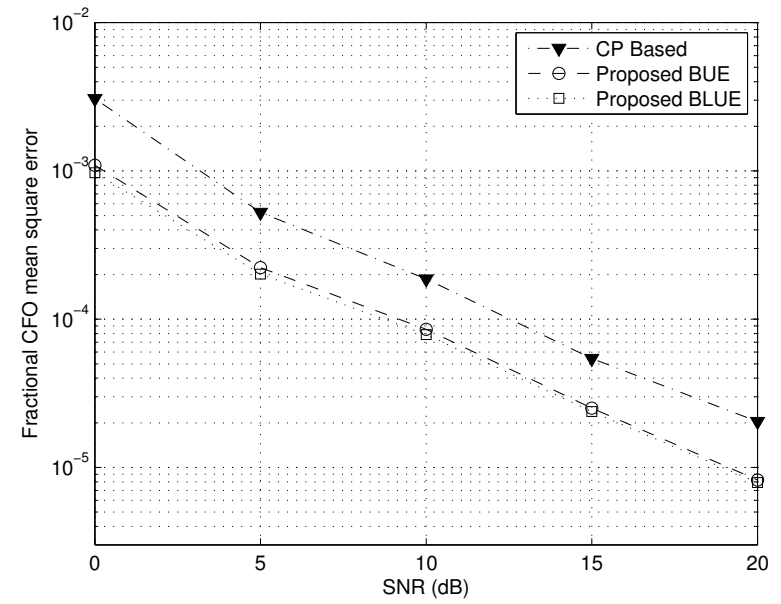

Fig. 3. Simulation results for IEEE 802.16 OFDMA physical layer $(M=3)$.

University. National ICT Australia is funded through the Australian Government's Backing Australia's Ability initiative and in part through the Australian Research Council.

\section{APPENDIX}

We derive the statistics of universal CFO estimation error in this section. Define $\gamma(d)$ as the sign of $\rho(d)$. Assume the estimation error is small, we can write

$$
\begin{aligned}
& \hat{\epsilon}(\hat{n}, d)-\left[\epsilon_{0}\right]_{1}=\frac{1}{2 \pi}\left(\arg (R[\hat{n}, d] R[\hat{n}, N-d])-2 \pi \epsilon_{0}\right) \\
= & \frac{1}{2 \pi}\left(\operatorname { a r g } \left(\arg \left(R[\hat{n}, N-d] \phi^{*}(N-d) \gamma(N-d)\right)\right.\right. \\
& \left.\left.\quad+R[\hat{n}, d] \phi^{*}(d) \gamma(d)\right)-2 \pi \epsilon_{0}\right) \\
= & \frac{1}{2 \pi}(\psi(d)+\psi(N-d))
\end{aligned}
$$

where

$$
\begin{aligned}
\psi(d) & \triangleq \arg \left(R[\hat{n}, d] \phi^{*}(d) \gamma(d) e^{-\frac{2 \pi}{N} d \epsilon_{0}}\right) \\
& =\tan ^{-1}\left(\frac{\Im\left(R[\hat{n}, d] \phi^{*}(d) \gamma(d) e^{-j \frac{2 \pi}{N} d \epsilon_{0}}\right)}{\Re\left(R[\hat{n}, d] \phi^{*}(d) \gamma(d) e^{-j \frac{2 \pi}{N} d \epsilon_{0}}\right)}\right) \\
& \approx \frac{\Im\left(\phi^{*}(d) \gamma(d) \Theta(\hat{n}, d)\right)}{(N-d)|\rho(d)| \sigma_{s}^{2}} .
\end{aligned}
$$

In $(21), \Re(\cdot)$ and $\Im(\cdot)$ denote the functions that take the real and imaginary parts of the argument respectively; the approximation follows the fact that for small $\theta, \tan ^{-1}(\theta) \approx \theta$. Because $\Theta(\hat{n}, d)$ has a random phase uniformly distributed in $[0,2 \pi)$,

$$
\begin{aligned}
E\left\{\hat{\epsilon}(\hat{n}, d)-\left[\epsilon_{0}\right]_{1}\right\} & =\frac{1}{2 \pi}\left(E\{\psi(d)\}+E\left\{\psi_{i}(N-d)\right\}\right) \\
& =0
\end{aligned}
$$

Denote $d_{1} \leq d_{2}$ as two correlation intervals. We can compute

$$
\begin{aligned}
& E\left\{\Theta^{*}\left(\hat{n}, d_{1}\right) \Theta\left(\hat{n}, d_{2}\right)\right\} \\
= & 2 \phi\left(d_{2}-d_{1}\right) \rho\left(d_{2}-d_{1}\right)\left(N-d_{2}\right) \sigma_{s}^{2} \sigma_{w}^{2} \\
& E\left\{\Theta\left(\hat{n}, d_{1}\right) \Theta\left(\hat{n}, d_{2}\right)\right\} \\
= & 2 \phi\left(d_{2}+d_{1}\right) \rho\left(d_{2}+d_{1}\right) \max \left(N-d_{1}-d_{2}, 0\right) \sigma_{s}^{2} \sigma_{w}^{2} .
\end{aligned}
$$

Thus, using the property that $\phi\left(d_{1}+d_{2}\right)=\phi\left(d_{1}\right) \phi_{i}\left(d_{2}\right)$, we have

$$
\begin{aligned}
& E\left\{\Im\left(\phi^{*}\left(d_{1}\right) \gamma\left(d_{1}\right) \Theta\left(\hat{n}, d_{1}\right)\right) \Im\left(\phi^{*}\left(d_{2}\right) \gamma\left(d_{2}\right) \Theta\left(\hat{n}, d_{2}\right)\right)\right\} \\
& =-\frac{\gamma\left(d_{1}\right) \gamma\left(d_{2}\right)}{4} E\left(\phi^{*}\left(d_{1}\right) \Theta\left(\hat{n}, d_{1}\right)-\phi\left(d_{1}\right) \Theta^{*}\left(\hat{n}, d_{1}\right)\right) \\
& \cdot\left(\phi^{*}\left(d_{2}\right) \Theta\left(\hat{n}, d_{2}\right)-\phi\left(d_{2}\right) \Theta^{*}\left(\hat{n}, d_{2}\right)\right) \\
& =\gamma\left(d_{1}\right) \gamma\left(d_{2}\right) \sigma_{s}^{2} \sigma_{w}^{2}\left(\rho\left(d_{2}-d_{1}\right)\left(N-d_{2}\right)\right. \\
& \left.-\max \left(N-d_{1}-d_{2}, 0\right) \rho\left(d_{2}+d_{1}\right)\right) \text {. }
\end{aligned}
$$

Therefore,

$$
\begin{aligned}
E\left\{\psi\left(d_{1}\right) \psi\left(d_{2}\right)\right\} & =\frac{\sigma_{w}^{2}}{\sigma_{s}^{2}} \frac{1}{\rho\left(d_{1}\right) \rho\left(d_{2}\right)}\left(\frac{\rho\left(d_{2}-d_{1}\right)}{N-d_{1}}\right. \\
& \left.+\frac{\rho\left(d_{2}+d_{1}\right) \max \left(N-d_{1}-d_{2}, 0\right)}{\left(N-d_{1}\right)\left(N-d_{2}\right)}\right) .
\end{aligned}
$$

Suppose $0<d_{1} \leq d_{2} \leq N / 2$, from (20) and (26), we attain

$$
\begin{aligned}
& E\left\{\left(\hat{\epsilon}\left(\hat{n}, d_{1}\right)-\left[\epsilon_{0}\right]_{1}\right)\left(\hat{\epsilon}\left(\hat{n}, d_{2}\right)-\left[\epsilon_{0}\right]_{1}\right)\right\} \\
= & \frac{1}{4 \pi^{2}} E\left\{\left(\psi\left(d_{1}\right)+\psi\left(N-d_{1}\right)\right)\left(\psi\left(d_{2}\right)+\psi\left(N-d_{2}\right)\right)\right\} \\
= & \frac{N}{4 \pi^{2}} \frac{\sigma_{w}^{2}}{\sigma_{s}^{2}} \frac{\rho\left(d_{2}-d_{1}\right)\left(N-d_{2}\right)+\rho\left(d_{2}+d_{1}\right) d_{2}}{\rho\left(d_{1}\right) \rho\left(d_{2}\right) d_{2}\left(N-d_{1}\right)\left(N-d_{2}\right)} .
\end{aligned}
$$

Thus, (18) immediately follows.

\section{REFERENCES}

[1] IEEE, "Air interface for fixed and mobile broadband wireless access systems," IEEE P802.16e/D12, Feb. 2005.

[2] T. M. Schmidl and D. C. Cox, "Robust frequency and timing synchronization for OFDM," IEEE Transactions on Communications, vol. 45, no. 12, pp. 1613-1621, Dec. 1997.

[3] A. J. Coulson, "Maximum likelihood synchronization for OFDM using a pilot symbol: algorithm," IEEE Journal on Selected Areas in Coтmиnications, vol. 19, no. 12, pp. 2486-2494, Dec. 2001.

[4] — - "Maximum likelihood synchronization for OFDM using a pilot symbol: analysis," IEEE Journal on Selected Areas in Communications, vol. 19 , no. 12 , pp. 2495-2503, Dec. 2001.

[5] M. Morelli and U. Mengali, "An improved frequency offset estimator for OFDM applications," IEEE Communications Letters, vol. 3, no. 3, pp. 75-77, Mar. 1999.

[6] H. Minn, P. Tarasak, and V. Bhargava, "OFDM frequency offset estimation based on BLUE principle," in Proc. IEEE Vehicular Technology Conference (VTC), vol. 2, Sep. 2002, pp. 24-28.

[7] T. Bhatt, V. Sundaramurthy, J. Zhang, and D. McCain, "Initial synchronization for 802.16e downlink," in Proc. the Fortieth Asilomar Conference on signals, systems and computers (ACSSC), Oct. 2006.

[8] M. Ruan, M. C. Reed, and Z. Shi, "Training symbol based coarse timing synchronization in OFDM systems," IEEE Transactions on Wireless Communications, vol. 8, no. 5, pp. 2558-2569, May 2009.

[9] M. Morelli, A. D'Andrea, and U. Mengali, "Frequency ambiguity resolution in OFDM systems," IEEE Communication Letters, vol. 4, no. 4, pp. 134-136, Apr. 2000.

[10] S. M. Kay, Fundamentals of statistical signal processing: estimation theory. Prentice Hall PTR, 1993.

[11] IEEE LAN/MAN Standards Committee, "Channel models for fixed wireless applications," Document IEEE 802.16.3c-01/29r4, 2003. 